\title{
Galaxy Luminosity Function: A New Analytic Expression
}

\author{
J. S. Alcaniz ${ }^{1,2}$ and J. A. S. Lima ${ }^{2}$ \\ ${ }^{1}$ Departamento de Física, Universidade Federal do Rio Grande do Norte, C.P. 1641, 59072-970, Natal-RN, Brasil \\ ${ }^{2}$ Instituto de Astronomia, Geofísica e Ciências Atmosféricas, Universidade de São Paulo, \\ Rua do Matão, 1226, Cidade Universitária, 05508-900, São Paulo, SP, Brazil
}

Received on 17 January, 2003

\begin{abstract}
We propose a new analytic approximation for the luminosity function of galaxies. The suggested expression behaves like the Schechter function at the faint end $\left(\phi \sim L^{\alpha}\right)$ but departs considerably at the bright end $\left(L>>L^{*}\right)$. We argue here that such a behavior may provide a better fit for the current observational data than does the Schechter function. Its practical interest is stressed by considering roughly the data set provided by the Stromlo-APM redshift survey. Implications on the estimates of the matter density parameter from mass-to-light ratio are also briefly discussed.
\end{abstract}

The galaxy luminosity function (GLF) is a key quantity for any study of galaxy evolution and one of the most important astrophysical tools in practical cosmology. It describes the distribution of galaxies with different intrinsic luminosities $L$ (or magnitudes $M$ ), and since the main source of data coming from the distant past is provided by the light emitted (or scattered) by ordinary matter, the GLF also supplies the primary information about the baryonic content of the Universe.

Over the years, many attempts have been done for determining the shape of the GLF (Zwicky 1957; Kiang 1961; Abell 1965; Arakelyan \& Kalloglyan 1975). Such studies culminated with an elegant analytic form proposed by Schechter (1976) which nowadays is usually referred to as the general Schechter luminosity function. In the introduction of his popular paper, Schechter also emphasized some of the main applications of the GLF (see also Felten 1977; 1985; Sandage et al. 1979; Binggeli et al. 1988). In the field of cosmology, this quantity is essential to determine evolutionary effects on the galaxy number counts (Loh \& Spillar 1986; Yoshi \& Takahara 1988); to analyze the spatial distribution of galaxies from redshift surveys (da Costa et al. 1988; Efstathiou et al. 1988; Loveday et al. 1992; Blanton et al. 2001); to constrain models of galaxy formation (Frenk et al. 1988); to study statistics of gravitational lenses (Fukugita \& Turner 1991; Kochanek 1996); and since almost all galaxy surveys are limited by apparent magnitude, an estimate of the GLF is also of fundamental importance for the analysis of the large-scale structure in the Universe (Efstathiou 1997). In the last few years, a considerable effort has also been invested in determining the shape of the GLF for different morphologies or spectral types as well as any dependence it may have on environment (Binggeli et al. 1988; Willmer 1997; Marzke et al. 1998; Christlein 2000; Madgwick et al. 2001).
The galaxy luminosity function, $\phi(L)$, is defined by the differential expression $d n(L)=\phi(L) d L$, where $d n(L)$ is the number of galaxies per unit volume with luminosity in the range $L$ to $L+d L$. The luminosity function of galaxies can be fitted by a Schechter-type distribution

$$
\phi(L) d L=\phi^{*}\left(\frac{L}{L^{*}}\right)^{\alpha} \exp \left(-\frac{L}{L^{*}}\right) d\left(\frac{L}{L^{*}}\right),
$$

where $L^{*}$ is the characteristic luminosity, $\alpha$ is an arbitrary pure number and $\phi^{*}$ is a normalization parameter whose dimension is the number density of galaxies. This expression has not been deduced from first principles, and as such, the free parameters must be determined from astronomical observations. As widely known, all these parameters are sensitive on the kind of galaxy being sampled.

Let us now consider the following luminosity distribution

$$
\phi(L) d L=\phi^{*}\left(\frac{L}{L^{*}}\right)^{\alpha}\left[1-(\eta-1) \frac{L}{L^{*}}\right]^{\frac{1}{\eta-1}} d\left(\frac{L}{L^{*}}\right),
$$

where $\eta$ is a new adjustable dimensionless parameter. This expression has been chosen so that in the limit $\eta \rightarrow 1$ the standard Schechter expression is recovered. Formally, this result follows directly from the known identity $\lim _{\mathrm{d} \rightarrow 0}(1+$ dy $)^{\frac{1}{d}}=\exp (y)$ (Abramowitz et al. 1972). Therefore, for generic values of $\eta \neq 1$, the new GLF is a product of two power laws instead of an exponential multiplied by a power law.

For values of $\eta>1$, the positiviness of the argument appearing in the second power factor of (2) defines naturally a maximum value for the absolute luminosity, namely $L_{\max }=L^{*} /(\eta-1)$. The existence of this cut-off implies that at the bright end the corresponding distribution (for each 

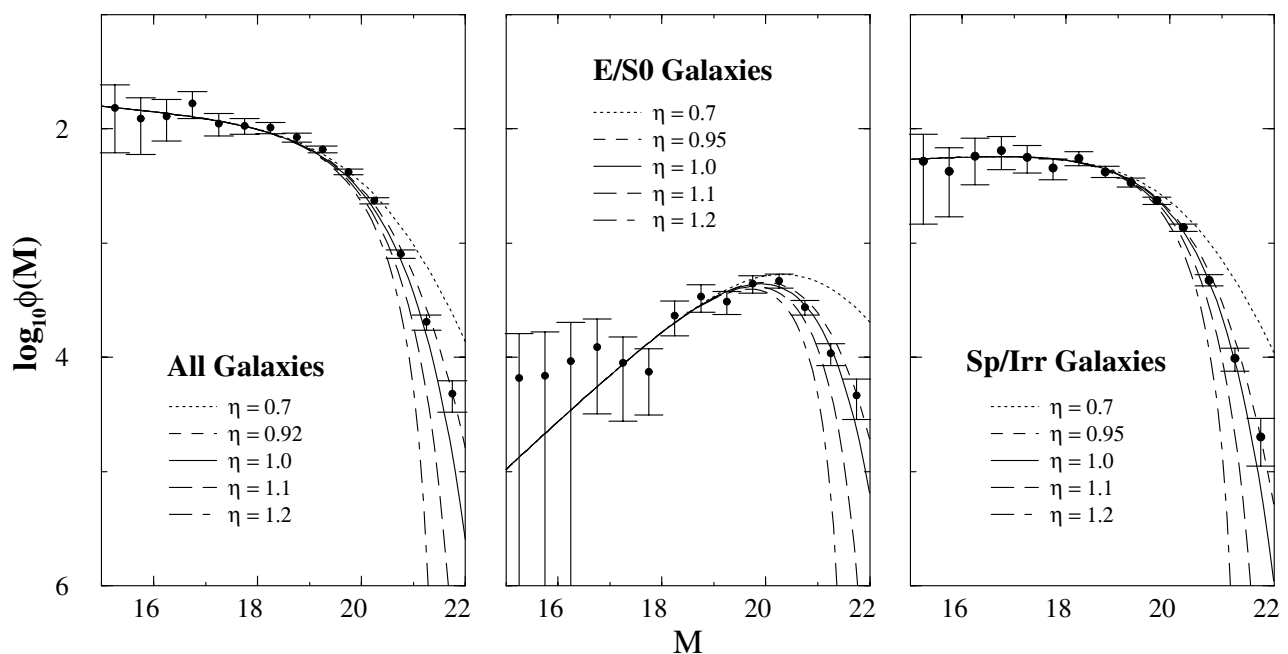

Figure 1. Luminosity function from the Stromlo-APM redshift survey. Fits in the three panels are to (a) the full galaxy sample, (b) the early-type subsample, and (c) the late-type galaxy subsample. The solid curves show Schechter function $(\eta=1)$. The dotted lines in each panel show how the fits change for the selected values of $\eta$ with the other parameter assuming the same values estimated in the quoted reference. In all these cases, the exponential Schechter function is a critical curve within a large class of possible power law distributions. Curves with $\eta>1$ fall off before the exponential while those with $\eta<1$ fall more slowly.

value of $\eta>1$ decreases faster than the standard Schechter function. However, in the complementary range $(\eta \leq 1)$ which which includes in particular the Schechter form for $\eta=1$, one may see that the cut-off is absent. The extended GLF approaches zero at the bright end more slowly than the Schechter distribution which is defined in terms of the exponential function. Note also that for low luminosities ( $L<<L^{*}$ ), the Schechter function and the new distribution present exactly the same behavior determined by the slope parameter $\alpha\left(\phi \sim L^{\alpha}\right)$. This means that the family of $\eta$-distributions proposed here essentially alters the behavior of the Schechter function at high luminosities. In other words, a given value of the slope parameter $\alpha$ can now be matched to a large class of asymptotic approaches to the bright end, which is described by a continuous parameter $\eta$. Conversely, for a given $\eta$ fitting the bright end of a given sample, the freedom on $\alpha$ still remains available at the faint end. In this way, the low and high- $L$ domains are now playing a more symmetrical role in the sense that both are free to be conveniently adjusted. It should be noticed that a "quasiSchechter" exponential behavior can be readily obtained for $\eta$ slightly different from unity.

In point of fact, astronomers prefer a magnitude representation for the GLF because $\phi(M)$ is more directly related to observations than $\phi(L)$. In terms of magnitude the new distribution reads:

$$
\begin{aligned}
\phi(M) d M & =0.921 \phi^{*} 10^{0.4\left(M^{*}-M\right)(\alpha+1)} \\
& \times\left[1-(\eta-1) 10^{0.4\left(M^{*}-M\right)}\right]^{\frac{1}{\eta-1}} d M
\end{aligned}
$$

where $M^{*}$ is a fiducial magnitude (defined by $L^{*}$ ) which yields the position of the knee appearing in the $\log \phi(M)$ curves whose inclinations (at the faint end) are determined by the possible values of $\alpha$. As an initial step, our aim here is to show that the proposed expression may be a more convenient approximation for the galaxy luminosity function than the usual Schechter function. In what follows, the features of this new function will be discussed more quantitatively in connection with the data set provided by the Stromlo-APM redshift survey (Loveday et al 1992).

In Fig. 1 we display the $\log _{10} \phi(M)-M$ plane based on the extended GLF for some selected values of the $\eta$ parameter corresponding to: (a) the full sample of galaxies, (b) the early-type subsample (E/SO), and (c) the late-type galaxy subsample ( $\mathrm{Sp} / \mathrm{Irr})$. In each picture, solid lines account for the best-fitting provided by a pure Schechter function $(\eta=1)$ whose parameters were determined using two different estimators by Loveday et al. (1992). The dotted lines show how the Schechter fit changes for the selected values of $\eta$ with the remaining parameters assuming the same values estimated in the quoted reference. In all these cases, the exponential Schechter function is a critical curve within a large class of possible power law distributions. Curves with $\eta>1$ fall off before the exponential while those with $\eta<1$ fall off more slowly. As remarked before, all curves coincide at low luminosities $\left(\phi \sim L^{\alpha}\right)$ regardless of the values assumed by the $\eta$ parameter $^{1}$.

At this point we analyze to what extent the $\eta$ parameter changes the estimates of the matter density in the Universe. This question is a natural one because the $\eta$ parameter appearing in the extended luminosity function modifies the mean luminosity density. It should be recalled that the relative contribution of the dark matter component in a given scale is usually specified by the $M / L$ ratio in the blue band.

\footnotetext{
${ }^{1}$ An analysis using the STY (Sandage et al. 1979) and SWML (Efstathiou et al. 1988) methods is currently being performed (Lima et al., in preparation).
} 
Mass-to-light ratio $\sim \mathbf{3 0 0 h}$

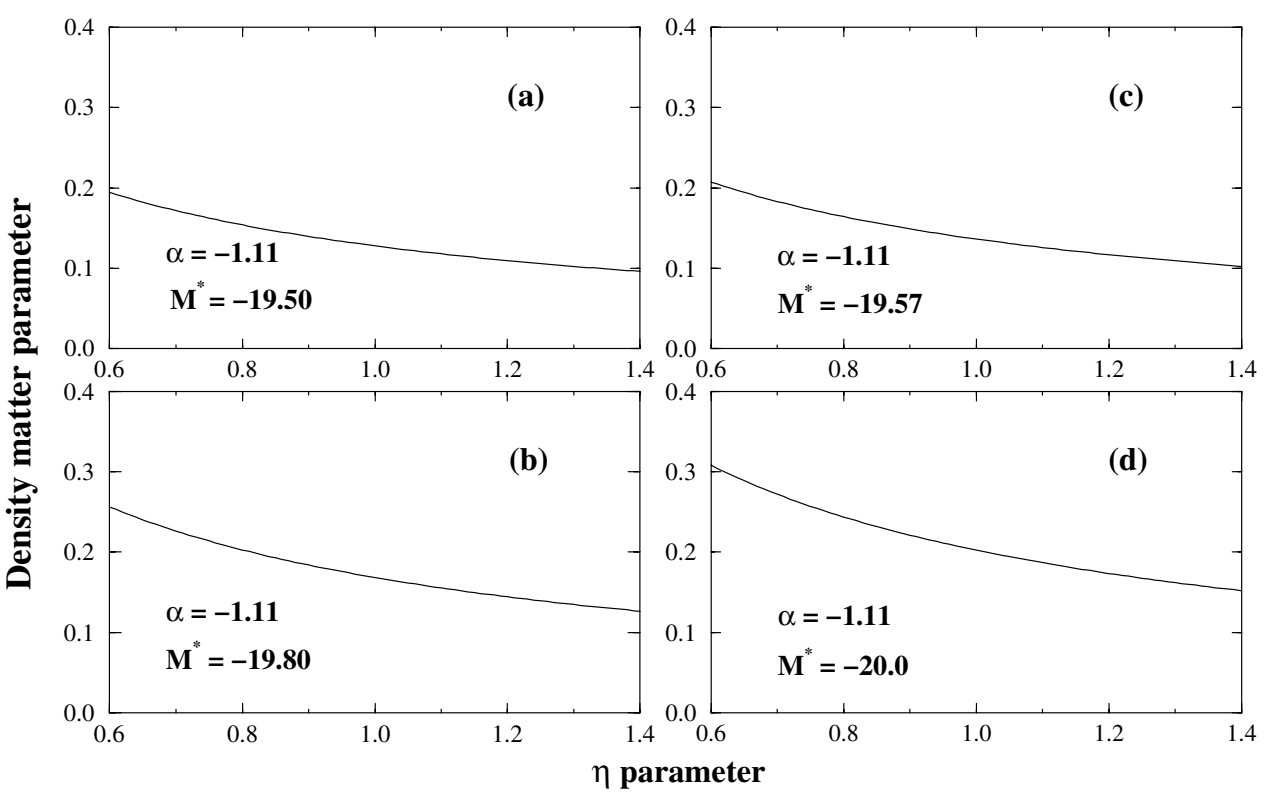

Figure 2. Mass-to-light ratio and the density parameter. The four panels show the dependence of $\Omega_{\mathrm{m}}$ as a function the index $\eta$ for some selected values of $M^{*}$. Note that in the four panels, the $\alpha$ parameter assumes a fixed value while $M^{*}$ is slightly varied. They show that for a fixed value of $\eta$ the value of $\Omega_{\mathrm{m}}$ increases with $M^{*}$. In all panels we see that smaller values of $\eta$ lead to greater values of $\Omega_{\mathrm{m}}$. This overall behavior holds regardless of the values assumed by the parameters $\alpha$ and $M^{*}$.

Typically, for the largest virialized systems (rich clusters of galaxies) this ratio (in solar units, $M_{\odot} / L_{\odot}$ ), falls on the interval $M / L \sim 300 \pm 100 h$ (Carlberg et al. 1996; Bachall 2000; Wilson et al. 2001). Parenthetically, this result still remains in agreement with the first measurements from the Coma cluster for which $M / L \sim 300 h$ (Zwick 1933), and seems to be rather independent of the velocity dispersion and other intrinsic parameters. Fixing this value for the mass-to-light ratio, $\Omega_{\mathrm{m}}$ can be estimated assuming that all the galaxies share the same $M / L$ ratio and that mass traces light on large scales. In this case, a subsequent integration over the observed luminosity density yields $\Omega_{\mathrm{m}} \simeq 0.2$ (Bachall 2000). When combined with the inflationary paradigm $\left(\Omega_{\text {Total }}=1\right)$, such a result means that nearly $80 \%$ of the mass in the Universe cannot be associated to the presence of galaxies. How is this result affected by the $\eta$ parameter?

In order to answer this question we first observe that for values of $\eta \geq 1$ and $\eta \leq 1$, the mean luminosities per unit volume are given, respectively, by

$$
\begin{aligned}
\mathcal{L}_{\eta \geq \infty} & =\int_{o}^{\mathrm{L}_{\max }} L \phi\left(\frac{L}{L^{*}}\right) d\left(\frac{L}{L^{*}}\right)= \\
& =\phi^{*} L^{*} \Gamma(\alpha+2) \frac{\Gamma\left(\frac{1}{\eta-1}\right)}{(\eta-1)^{\alpha+3} \Gamma\left(\alpha+3+\frac{1}{\eta-1}\right)},
\end{aligned}
$$

$$
\begin{aligned}
\mathcal{L}_{\eta \leq \infty} & =\int_{o}^{\infty} L \phi\left(\frac{L}{L^{*}}\right) d\left(\frac{L}{L^{*}}\right)= \\
& =\phi^{*} L^{*} \Gamma(\alpha+2) \frac{\Gamma\left(\frac{1}{1-\eta}-\alpha-2\right)}{(1-\eta)^{\alpha+2} \Gamma\left(\frac{1}{1-\eta}\right)}
\end{aligned}
$$

where $\Gamma$ is the usual gamma function. As one may check, by considering the identity $\lim _{|\mathrm{z}| \rightarrow \infty} \mathrm{z}^{-\mathrm{a}} \Gamma(\mathrm{a}+\mathrm{z}) / \Gamma(\mathrm{z})=1$ (Abramowitz et al. 1972), the standard result $\mathcal{L}=\Gamma(\alpha+$ 2) $\phi^{*} L^{*}$ (see, for instance, Peebles 1993) is readily recovered as a limiting case of the above expressions $(\eta \rightarrow 1)$. Thus, since the critical mass density of the Einstein-de Sitter model is $\rho_{\text {crit }}=2.78 \times 10^{11} h^{2} M_{\odot} \mathrm{Mpc}^{-3}$, the mass-tolight ratio required to close the Universe can easily be found by combining $\rho_{\text {crit }}$ and $\mathcal{L}$.

In Fig. 2 we fix $M / L=300 h M_{\odot} / L_{\odot}$ (Bahcall 2000) and $\phi^{*}=1.12 \times 10^{-2} h \mathrm{Mpc}^{-3}$ (Loveday et al. 1992) and display $\Omega_{\mathrm{m}}$ as a function of the index $\eta$. In the four panels, the parameter $\alpha$ has been fixed by the value obtained for the pure Schechter function while $M^{*}$ is slightly varied. We see that for a fixed value of $\eta$ the value of $\Omega_{\mathrm{m}}$ increases with $M^{*}$. For instance, for $\eta=0.91$ and $M^{*}=-19.50$ we find $\Omega_{\mathrm{m}} \simeq 0.13$ whereas for the same value of $\eta$ and $M^{*}=-19.80$ we have $\Omega_{\mathrm{m}} \simeq 0.19$. As a general behavior we see that the smaller the values of $\eta$ the greater the values of $\Omega_{\mathrm{m}}$.

Finally, we stress that the consistency of the phenomenological parameters appearing in the luminosity function should be tested using many different methods. In particular, for the exponential Schechter function, this aspect has been analyzed by using several independent treatments (see, for instance, Willmer 1997). In this concern, it should be interesting to investigate the goodness of the fit for these data provided by the extended luminosity function proposed here. Such an analysis including the computation of the 
shape parameters will appear in a forthcoming communication.

\section{Acknowledgments}

This work was partially suported by the Conselho Nacional de Desenvolvimento Científico e Tecnológico (CNPq), CNPq (62.0053/01-1-PADCT III/Milenio), Pronex/FINEP (no. 41.96.0908.00) and FAPESP (00/06695-0)

\section{References}

[1] G.O. Abell, ARA\&A, 3, 1 (1965).

[2] M. Abramowitz \& I. A. Stegun Handbook of Mathematical Functions, Dover, NY, 1972.

[3] M.A. Arakelyan \& A.T. Kalloglyan, Soviet Astr. Astron. 13, 953 (1975).

[4] N.A. Bahcall, Phys. Rep 333, 233 (2000).

[5] B. Binggeli, A. Sandage, \& G.A. Tammann ARA\&A, 26, 509 (1988).

[6] M.R. Blanton et al., Astron. J. 121, 2358 (2001).

[7] R.G. Calberg et al., Astrop. J. 462, 32 (1996).

[8] D. Christlein, Astrop. J. 545, 145 (2000).

[9] L.N. da Costa, P.S. Pellegrini, W.L.W. Sargent, J. Tonry, M. Davis, A. Meiksin, \& D.W. Latham, Astrop. J. 327, 544 (1988)

[10] G. Efstathiou, in Cosmologie et structure à grande échelle, Eds. R. Schaeffer, J. Silk, M. Spiro, \& J. Zinn-Justin, Elsevier, 133, 1997.
[11] G. Efstathiou, R.S. Ellis, \& B.A. Peterson MNRAS, 232, 431 (1988).

[12] J.E. Felten, Astron. J. 82, 861 (1977).

[13] J.E. Felten, Comm. Astrophys. 11, 53 (1985).

[14] C.S. Frenk et al., Astrop. J. 327, 507 (1988).

[15] M. Fukugita \& E.L. Turner, MNRAS 253, 99 (1991).

[16] T.D. Kiang, MNRAS, 122, 263 (1961).

[17] C. Kochaneck, Astrop. J. 466, 638 (1996).

[18] J.A.S. Lima et al. 2003, in preparation

[19] E. Loh \& E. Spillar, Astrop. J. 307, L1 (1986).

[20] J. Loveday, B.A. Peterson, G. Efstathiou, \& S.J. Maddox, Astrop. J. 390, 338 (1992).

[21] Madgwick et al. 2001, MNRAS, in press, astro-ph/0107197

[22] R.O. Marzke, L.N. da Costa, P.S. Pellegrini, C.N.A. Willmer, \& M.J. Geller, Astrop. J. 503, 617 (1998).

[23] P.J.E. Peebles. 1993, Principles of Physical Cosmology, Princeton UP, 1993.

[24] A. Sandage, G.A. Tamman, \& A. Yahil, Astrop. J. 232, 352 (1979).

[25] P.L. Schechter, Astrop. J. 203, 297 (1976).

[26] C.N.A. Willmer, Astron. J. 114, 898 (1997).

[27] G. Wilson, N. Kaiser \& G.A. Luppino, Astrop. J. 556, 601 (2001).

[28] Y. Yoshii \& F. Takahara, Astrop. J. 326, 1 (1988).

[29] F. Zwicky, Morphological Astronomy, Berlin: SpringerVerlag, 1957, 